\title{
Asteroseismology of fast-rotating stars: the example of $\alpha$ Ophiuchi
}

\author{
Giovanni M. Mirouh ${ }^{1,2}$, Daniel R. Reese ${ }^{3}$, Francisco Espinosa Lara ${ }^{1,2}$, \\ Jérôme Ballot ${ }^{1,2}$ and Michel Rieutord ${ }^{1,2}$ \\ ${ }^{1}$ Université de Toulouse, UPS-OMP, IRAP, Toulouse, France \\ ${ }^{2}$ CNRS, IRAP, 14 avenue Edouard Belin, 31400 Toulouse, France \\ ${ }^{3}$ Institut d'Astrophysique et Géophysique de l'Université de Liège, Allée du 6 Août 17, \\ 4000 Liège, Belgium
}

\begin{abstract}
Many early-type stars have been measured with high angular velocities. In such stars, mode identification is difficult as the effects of fast and differential rotation are not well known. Using fundamental parameters measured by interferometry, the ESTER structure code and the TOP oscillation code, we investigate the oscillation spectrum of $\alpha$ Ophiuchi, for which observations by the MOST satellite found 57 oscillations frequencies. Results do not show a clear identification of the modes and highlight the difficulties of asteroseismology for such stars with a very complex oscillation spectrum.
\end{abstract}

Keywords. stars: oscillations, stars: rotation, stars: individual: $\alpha$ Oph, Rasalhague

\section{Introduction}

Intermediate- and high-mass stars are usually fast rotators. In some of these A-, B- and O-type stars, the $\kappa$ mechanism excites eigenmodes. Because of the centrifugal flattening of the star and the strength of the Coriolis acceleration, the oscillation spectrum is much more complex than that of non-rotating stars. Interpretation of observed frequencies of these stars requires two-dimensional models and their oscillation spectrum computed in a non-perturbative way. Following the pioneering work of Deupree et al. (2012), we want to identify the observed modes of the fast-rotating star $\alpha$ Ophiuchi in order to better constrain its fundamental parameters. Compared to the previous work of Deupree, we improve the numerical resolution and get spectrally converged eigenfunctions that allow a reliable computation of visibilities and damping rates of the modes.

\section{Models}

We compute 2D models for the fast-rotating A-type star Rasalhague ( $\alpha$ Ophiuchi) with the ESTER code (Rieutord \& Espinosa Lara 2013), in which differential rotation is calculated self-consistently. Its surface equatorial rotation velocity of $240 \mathrm{~km} \mathrm{~s}^{-1}$ imposes a flatness of 0.168 . Its equatorial and polar radii have been derived from interferometry (Zhao et al. 2009) and the stellar mass is constrained by an orbiting companion (Hinkley et al. 2011). Besides this, 57 oscillation frequencies have been measured by photometry (Monnier et al. 2010). To reproduce the fitted radii, luminosity, polar and equatorial temperatures, our model uses $M=2.22 M_{\odot}, \Omega=0.62 \Omega_{\mathrm{K}}=1.65 \mathrm{~d}^{-1}$, mass fractions $Z=0.02$ and $X=0.7$ in the envelope, $X_{\mathrm{c}}=0.26$ in the core (see Espinosa Lara \& Rieutord 2013). The eigenvalue problem of adiabatic oscillations is solved with the TOP code (Reese et al. 2009) for modes with azimuthal orders $-4 \leqslant m \leqslant 4$, in the range of frequencies in which the p modes are thought to be observed (see Fig. 1). 

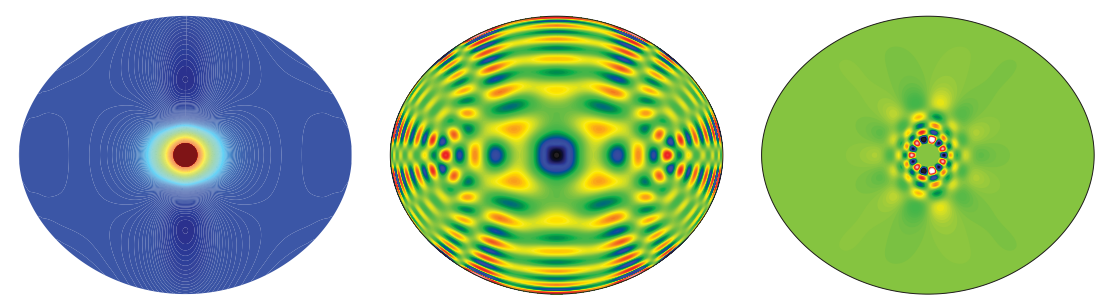

Figure 1. From left to right: Rotation profile in $\alpha$ Oph (fast core, slow envelope), an $m=0$ acoustic island-mode, an $m=-1$ g-mode.

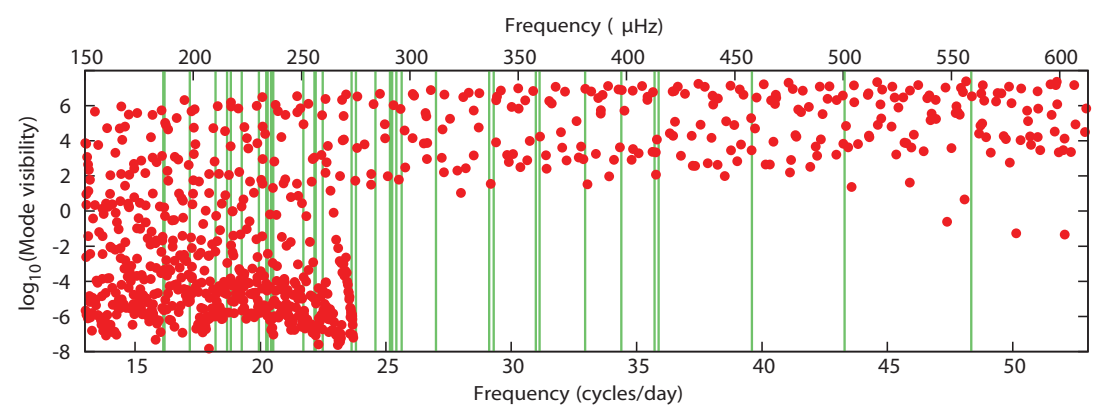

Figure 2. Visibilities for axisymmetric $(m=0)$ modes. The vertical lines indicate the frequencies measured with MOST (Monnier et al. 2010).

\section{Comparisons between computed and observed modes}

To select the modes that might be seen from Earth, we compute the mode visibilities, following Reese et al. (2013) and the thermal dissipation rates using the quasi-adiabatic approximation (Unno et al. 1989). In the domain that we investigated, our model yields only linearly stable modes. These are g modes and $\mathrm{p}$ modes modified by rotation. The $\mathrm{g}$ modes are the least-damped with large amplitude at the base of the envelope. Their visibility is much less than that of the $\mathrm{p}$ modes that exist in this interval, as their amplitude is evanescent at the surface. However, p modes are much more damped. As shown in Fig. 2, where we clearly distinguish the set of g modes on the low-frequency side, each observed frequency corresponds to several eigenmodes of the model. The damping rates do not seem to be able to lift this degeneracy of the matching. It may well be that our models are at the moment too simple, notably because of the chemical homogeneity of the envelope. Progress therefore calls for more realistic models that include the distribution of chemical elements resulting from time evolution.

\section{References}

Deupree, R. G., Castañeda, D., Peña, F., \& Short, C. I. 2012, ApJ, 753, 20

Espinosa Lara, F. \& Rieutord, M. 2013, A\&A, 552, A35

Hinkley, S., Monnier, J. D., Oppenheimer, B. R., et al. 2011, ApJ, 726, 104

Monnier, J. D., Townsend, R. H. D., Che, X., et al. 2010, ApJ, 725, 1192

Reese, D. R., MacGregor, K. B., Jackson, S., et al. 2009, A\&A, 506, A189

Reese, D. R., Prat, V., Barban, C., et al. 2013, A\&A, 550, A77

Rieutord, M. \& Espinosa Lara, F. 2013, in: Lecture Notes in Physics, vol. 865, p. 49

Unno, W., Osaki, Y., Ando, H., Saio, H., \& Shibahashi, H. 1989, Nonradial oscillations of stars (Univ. of Tokyo Press: Tokyo)

Zhao, M., Monnier, J. D., Pedretti, E., et al. 2009, ApJ, 701, 209 